\title{
A SPATIAL WEB GRAPH MODEL WITH LOCAL INFLUENCE REGIONS
}

\author{
W. AIELLO, A. BONATO, C. COOPER, J. JANSSEN, AND P. PRAŁAT
}

\begin{abstract}
We present a new stochastic model for complex networks, based on a spatial embedding of the nodes, called the Spatial Preferred Attachment (SPA) model. In the SPA model, nodes have influence regions of varying size, and new nodes may only link to a node if they fall within its influence region. The spatial embedding of the nodes models the background knowledge or identity of the node, which will influence its link environment. In our model, nodes can determine their link environment based only on local knowledge of the network. We prove that our model gives a power law in-degree distribution, with exponent in $[2, \infty)$ depending on the parameters, and with concentration for a wide range of in-degree values. We show that the model allows for edges that span a large distance in the underlying space, modelling a feature often observed in real-world complex networks.
\end{abstract}

\section{INTRODUCTION}

Current stochastic models for complex networks, such as those described in [2, 3], aim to reproduce a number of graph properties observed in real-world networks such as the web graph. On the other hand, experimental and heuristic treatments of real-life networks operate under the tacit assumption that the network is a visible manifestation of an underlying hidden reality. For example, it is commonly assumed that communities in a social network can be recognized as densely linked subgraphs, or that web pages with many common neighbours contain related topics. Such assumptions imply that there is an a priori community structure or relatedness measure of the nodes, which is reflected by the link structure of the graph.

A common method to represent relatedness of objects is by an embedding in a metric space, so that related objects are placed close together, and communities are represented by clusters of points. Following a common text mining technique, web pages are often represented as vectors in a word-document space. Using Latent Sematic Indexing, these vectors can then be embedded in a Euclidean topic space, so that pages on similar topics are located close together. Experimental studies [8] have confirmed that similar pages are more likely to link to each other. On the other hand, experiments also confirm a large amount of topic drift: it is possible to move to a completely different topic in a relatively short number of hops. This points to a model where nodes are embedded in a metric space, and the edge probability between nodes is influenced by their proximity, but edges that span a larger distance in the space are not uncommon.

The Spatial Preferred Attachment (SPA) model proposed in this paper combines the above considerations with the often-used preferential attachment principle: pages with

The authors gratefully acknowledge support from NSERC and MITACS grants. 
high in-degree are more likely to receive new links. In the SPA model, each node is placed in space and surrounded by an influence region. The volume of the influence region is determined by the in-degree of the node. The volume of each region is scaled by time, so the influence regions of nodes that do not gain new links will steadily decrease in size. The decrease in the volume of influence regions is motivated by the fact that topics space grows over time. A new node $v$ can only link to an existing node $u$ if $v$ falls within the influence region of $u$. If $v$ falls within the influence region of $u$, then $v$ will link to $u$ with probability $p$. Thus, the model is based on the preferential attachment principle, but only implicitly: nodes with high in-degree have a large influence region, and therefore are more likely to attract new links.

A random graph model with certain similarities to the SPA model is the geometric random graph; see [9]. In that model, all influence regions have the same size, and the link probability is $p=1$. Flaxman, Frieze, and Vera [6] supply an interesting geometric model where nodes are embedded on a sphere, and the link probability is influenced by the relative positions of the nodes. This model is a generalization of a geometric preferential attachment models presented by the same authors in [5], which influenced our model.

There are at least three features that distinguish the SPA model from previous models. First, a new node can choose its links purely based on local information. Namely, the influence region of a node can be seen as the region where the associated entity (such as a web page or scholarly paper) is visible: only entities that are close enough (in topic) to fall within the influence region will be aware of its existence, and thus have a possibility to link to it. Moreover, a new node links independently to each node visible to it. Consequently, the new node needs no knowledge of the invisible part of the graph (such as in-degree of other nodes, or total number of nodes or links) to determine its neighbourhood. Second, since a new node links to each visible node independently, the out-degree is not a constant nor chosen according to a pre-determined distribution, but arises naturally from the model. Third, the varying size of the influence regions allows for the occasional long links, edges between nodes that are spaced far apart. This implies a certain "small world" property.

1.1. The SPA model. We formally define the SPA model as follows. Fix parameters $m \in \mathbb{N}$, the dimension, and $p \in[0,1]$, the link probability. In addition, fix three positive constants $A_{1}, A_{2}$ and $A_{3}$ so that $p A_{1} \leq 1$. Let $S$ be the unit hypercube in $\mathbb{R}^{m}$, with the torus metric $d(\cdot, \cdot)$ derived from the $L_{\infty}$ metric. In particular, for any two points $x$ and $y$ in $\mathbb{R}^{m}$,

$$
d(x, y)=\min \left\{\|x-y+u\|_{\infty}: u \in\{-1,0,1\}^{n}\right\} .
$$

The torus metric is chosen so that there are no boundary effects, and altering the metric will not significantly affect the main results of the paper. The $L_{\infty}$ norm is chosen so that every point on the boundary of the unit cube has equal distance $1 / 2$ to the center of the hypercube. However, the norm could be easily replaced by any of the $L_{p}$ norms, with changes to only constants in our main results.

For each positive real number $\alpha \leq 1$, and $u \in S$, define the ball around $u$ with volume $\alpha$ as

$$
B_{\alpha}(u)=\left\{x \in S: d(u, x) \leq r_{\alpha}\right\},
$$


where $r_{\alpha}=\alpha^{1 / m} / 2$, so $r_{\alpha}$ is chosen such that $B_{\alpha}$ has volume $\alpha$.

The SPA model generates stochastic sequences of graphs $\left(G_{t}: t \geq 0\right)$, where $G_{t}=$ $\left(V_{t}, E_{t}\right)$, and $V_{t} \subseteq S$. Let $d^{-}(v, t)$ be the in-degree of node $v$ in $G_{t}$, and $d^{+}(v, t)$ its out-degree. We define the influence region of node $v$ at time $t \geq 1$, written $R(v, t)$, to be the ball around $v$ with volume

$$
|R(v, t)|=\frac{A_{1} d^{-}(v, t)+A_{2}}{t+A_{3}},
$$

or $R(v, t)=S$ if the right-hand-side is greater than 1 .

The process begins at $t=0$, with $G_{0}$ being the empty graph. Time-step $t, t \geq 1$, is defined to be the transition between $G_{t-1}$ and $G_{t}$. At the beginning of each time-step $t$, a new node $v_{t}$ is chosen uniformly at random (uar) from $S$, and added to $V_{t-1}$ to create $V_{t}$. Next, independently, for each node $u \in V_{t-1}$ such that $v_{t} \in R(u, t-1)$, a directed edge $\left(v_{t}, u\right)$ is created with probability $p$. Thus, the probability that a link $\left(v_{t}, u\right)$ is added in time-step $t$ equals $p|R(u, t-1)|$.

Because new nodes choose independently whether to link to each visible node, and the size of the influence region of a node depends only on the edges from younger nodes, the distribution of the random graph $G_{n}$ produced by the SPA model with parameters $A_{1}, A_{2}, A_{3}, p, m$ is equivalent to the graph $G_{n+A_{3}}$ produced by the SPA model with the same values for $A_{1}, A_{2}, p, m$, but with $A_{3}=0$, where the first $A_{3}$ nodes have been removed. Since the results presented in this paper do not depend on the first nodes, we will assume throughout that $A_{3}=0$. In the rest of the paper, $\left(G_{t}: t \geq 0\right)$ refers to a sequence of random graphs generated by the SPA model with parameters $A_{1}, A_{2}, p$, and $m$, and we assume $A_{3}=0$. We use the notation $[n]$ for $\{0,1, \ldots, n\}$. All logarithms are in base $e$.

1.2. Main Results. We now state our main results on the SPA model, with proofs deferred to the next section. We first prove that with high probability a graph $G_{n}$ generated by the SPA model has an in-degree distribution that follows a power law. We say that an event holds asymptotically almost surely (aas) if it holds with probability tending to one as $n \rightarrow \infty$. An event holds with extreme probability (wep) if it holds with probability at least $1-\exp \left(-\Theta\left(\log ^{2} n\right)\right)$ as $n \rightarrow \infty$. We will often use the stronger notion of wep in favour of the more commonly used aas, since it simplifies some of our proofs. If we consider a polynomial number of events that each holds wep, then wep all events hold. Let $N_{i, t}$ denote the number of nodes of in-degree $i$ in $G_{t}$. For an integer $n \geq 0$, define

$$
i_{f}=i_{f}(n)=\left(\frac{n}{\log ^{8} n}\right)^{\frac{p A_{1}}{4 p A_{1}+2}} .
$$

Theorem 1.1. Fix $p \in(0,1]$. Then for any $i \geq 0$,

$$
\mathbb{E}\left(N_{i, n}\right)=(1+o(1)) c_{i} n,
$$

where

$$
c_{0}=\frac{1}{1+p A_{2}}
$$


and for $1 \leq i \leq n$,

$$
c_{i}=\frac{p^{i}}{1+p A_{2}+i p A_{1}} \prod_{j=0}^{i-1} \frac{j A_{1}+A_{2}}{1+p A_{2}+j p A_{1}} .
$$

For $i=0, \ldots, i_{f}$, wep

$$
N_{i, n}=(1+o(1)) c_{i} n
$$

Since $c_{i}=(1+o(1)) c i^{-\left(1+\frac{1}{p A_{1}}\right)}$ for some constant $c$, this shows that for large $i$, the expected proportion $N_{i, n} / n$ follows a power law with exponent $1+\frac{1}{p A_{1}}$, with concentration for all values of $i$ up to $i_{f}$. If $p A_{1}=10 / 11$, then the power law in-degree exponent is 2.1 , the same as observed in the web graph (see $[2,3])$.

The previous result characterizes the distribution of in-degrees in the graph. The total number of nodes of a given in-degree (smaller than $i_{f}$ ) is tightly concentrated around its mean. In the next result, we give a precise expression for the probability distribution of the in-degree of the individual node $v_{i}$ born at time $i$, in the case that $p A_{1}<1$. No concentration result can be obtained here, but part (c) does give a bound on the maximum value that the in-degree of any particular vertex can reach.

Let $v_{j}$ be the node added at time-step $j$ and let $d^{-}\left(v_{j}, n\right)$ be the in-degree of this node at the end of time-step $n$.

Theorem 1.2. If $0<p A_{1}<1$, then the following hold.

(a) For $1 \leq j \leq n\left(1-\log ^{-1} n\right)$ and $0 \leq l \leq \sqrt{j} \log ^{-1} n$ or for $n\left(1-\log ^{-1} n\right)<j<n$ and $l=0,1$,

$\mathbb{P}\left(d^{-}\left(v_{j}, n\right)=l\right)=\left(1+O\left(\log ^{-1} n\right)\right)\left(\begin{array}{c}l+\left(A_{2} / A_{1}\right)-1 \\ l\end{array}\right)\left(\frac{j}{n}\right)^{p A_{2}}\left(1-\left(\frac{j}{n}\right)^{p A_{1}}\left(1+O\left(\log ^{-1} n\right)\right)\right)^{l}$.

(b) For $n\left(1-\log ^{-1} n\right)<j<n$ and $l \geq 2$,

$$
\mathbb{P}\left(d^{-}\left(v_{j}, n\right)=l\right)=O\left(l^{\left(A_{2} / A_{1}\right)-1} / \omega^{l}\right) .
$$

(c) For all $K>0$,

$$
\mathbb{P}\left(\text { There exists } j \leq n: d^{-}\left(v_{j}, n\right) \geq K \omega^{2}(n / j)^{p A_{1}}\right)=O\left(n^{-K e^{-18}}\right) .
$$

Theorem 1.2(c) implies (taking $K=\log ^{2} n$ ) that wep every node $v_{j}$ has in-degree at most $(n / j)^{p A_{1}} \log ^{4} n$. Conditional on this, items (a) and (b) characterize the distribution of $d^{-}\left(v_{j}, n\right)$ for all $j \geq \log ^{8} n$ when $p A_{1} \leq 1 / 2$ and for $j \geq n^{p A_{1}-1 / 2} \log ^{8} n$ when $p A_{1}>1 / 2$.

Let $M_{t}=\left|E_{t}\right|$, the number of edges in $G_{t}$, and let $m_{t}=\mathbb{E}\left(M_{t}\right)$. Then we have that

$$
\mathbb{E}\left(M_{t+1} \mid M_{t}\right)=M_{t}+\sum_{j=1}^{t} p \frac{A_{1} d^{-}\left(v_{j}, t\right)+A_{2}}{t}=M_{t}+\frac{p A_{1} M_{t}}{t}+p A_{2},
$$

and so $m_{1}=0$, and for $t \geq 1$,

$$
m_{t+1}=m_{t}\left(1+\frac{p A_{1}}{t}\right)+p A_{2}
$$


The (first-order) solutions of this recurrence are

$$
m_{n} \sim \begin{cases}\frac{p A_{2}}{1-p A_{1}} n, & p A_{1}<1 \\ n \log n, & p A_{1}=1 .\end{cases}
$$

Theorem 1.3. If $p A_{1}<1$, then aas the number of edges is concentrated around its expected value:

$$
M_{n}=(1+o(1)) m_{n}
$$

An important difference between the SPA model and many other models is that the out-degree is not a parameter of the model, but is the result of a stochastic process. Using the expression for $m_{n}$ above, we can easily derive the expected out-degree of a vertex $v_{j}$. For example, this out-degree equals $p A_{2} /\left(1-p A_{1}\right)+o(1)$ if $p A_{1}<1$. Since the expected out-degree is small, we do not expect concentration. The next result gives bounds for the maximum out-degree in the graph.

Theorem 1.4. Aas

$$
\max _{0 \leq i \leq n} \operatorname{deg}^{+}\left(v_{i}, n\right) \geq(1+o(1)) p \frac{\log n}{\log \log n} .
$$

However, aas all nodes have out-degree $O\left(\log ^{2} n\right)$.

Theorem 1.5. Aas $\operatorname{deg}^{+}\left(v_{n}, n\right)=O\left(\log ^{2} n\right)$.

From Theorem 1.2, the number of nodes in a graph generated by the SPA model of in-degree zero in $G_{n}$ is linear in $n$. In addition, with positive probability a new node will land in a part of $S$ not covered by any influence regions, and thus, have out-degree zero. Therefore, the underlying undirected graph of $G_{n}$ is not connected. In fact, we expect that for the majority of distinct pairs $u, v$, there will not be a directed path from $u$ to $v$. Since this is a property also observed in the web graph, it does not detract from the SPA model, but rather indicates that we should consider another variable rather than diameter to indicate a "small world" property. Thus, we focus on the (geometric) distance, in $S$, spanned by the links.

For a pair of points $u, v \in S$. let $L(u, v)$ be the length of the shortest curve embedded in the surface of $S$ that connects $u$ and $v$. Define

$$
L_{t}=\sum_{\left(v_{t}, v_{i}\right) \in E_{t}} L\left(v_{t}, v_{i}\right)
$$

that is, $L_{t}$ is the sum of the lengths of new edges added at time $t$ in the SPA model. Note that $L_{t}$ is a continuous random variable.

Theorem 1.6. For the expectation of $L_{t}$, we have that

$$
\mathbb{E}\left(L_{t}\right)= \begin{cases}\Theta\left(t^{-} \min \{1 / m)\right. & \text { if } 1 / m<1 /\left(p A_{1}\right)-1 \\ \Theta\left(t^{-} \min \{1 / m \log n)\right. & \text { if } 1 / m=1 /\left(p A_{1}\right)-1 \\ \Theta\left(t^{-} \min \{1 / m)\right. & \text { if } 1 / m>1 /\left(p A_{1}\right)-1\end{cases}
$$


Theorem 1.6 contrasts with the analogous result for graphs generated with a similar process to the SPA model, but where all influence regions have volume $d / t$ for $d>$ 0 a constant. We call this the egalitarian model. In the egalitarian model, $\mathbb{E}\left(L_{t}\right)$ decreases much faster with $t$ than for the SPA model if $p A_{1}$ is large, specifically when $p A_{1}>\frac{m}{m+1}$. For example, if $p A_{1}=1$, then $\mathbb{E}\left(L_{t}\right)=\Theta(1)$ for the SPA model, while $\mathbb{E}\left(L_{t}\right)=\Theta\left(t^{m /(m+1)}\right)$ for the egalitarian model.

Theorem 1.7. In the egalitarian model with influence regions of volume $d / t$ for $d>0$ a constant, we have that

$$
\mathbb{E}\left(L_{t}\right)=\Theta\left(t^{-1 / m}\right)
$$

\section{Proofs of Results}

This section is devoted to the proofs of the theorems outline in the previous section.

2.1. Proof of Theorem 1.1. The equations relating the random variables $N_{i, t}$ are described as follows. As $G_{1}$ consist of one isolated node, $N_{0,1}=1$, and $N_{i, 1}=0$ for $i>0$. For all $t>0$, we derive that

$$
\begin{aligned}
\mathbb{E}\left(N_{0, t+1}-N_{0, t} \mid G_{t}\right) & =1-N_{0, t} p \frac{A_{2}}{t}, \\
\mathbb{E}\left(N_{i, t+1}-N_{i, t} \mid G_{t}\right) & =N_{i-1, t} p \frac{A_{1}(i-1)+A_{2}}{t}-p N_{i, t} \frac{A_{1} i+A_{2}}{t} .
\end{aligned}
$$

Recurrence relations for the expected values of $N_{i, t}$ can be derived by taking the expectation of the above equations. To solve these relations, we use the following lemma on real sequences, which is Lemma 3.1 from [3].

Lemma 2.1. If $\left(\alpha_{t}\right),\left(\beta_{t}\right)$ and $\left(\gamma_{t}\right)$ are real sequences satisfying the relation

$$
\alpha_{t+1}=\left(1-\frac{\beta_{t}}{t}\right) \alpha_{t}+\gamma_{t}
$$

and $\lim _{t \rightarrow \infty} \beta_{t}=\beta>0$ and $\lim _{t \rightarrow \infty} \gamma_{t}=\gamma$, then $\lim _{t \rightarrow \infty} \frac{\alpha_{t}}{t}$ exists and equals $\frac{\gamma}{1+\beta}$.

Applying this lemma with $\alpha_{t}=\mathbb{E}\left(N_{0, t}\right), \beta_{t}=p A_{2}$, and $\gamma_{t}=1$ gives that $\mathbb{E}\left(N_{0, t}\right)=$ $c_{0} t+o(t)$ with $c_{0}$ as in (1). For $i>0$, the lemma can be inductively applied with $\alpha_{t}=$ $\mathbb{E}\left(N_{i, t}\right), \beta_{t}=p\left(A_{1} i+A_{2}\right)$, and $\gamma_{t}=\mathbb{E}\left(N_{i-1, t}\right) \frac{A_{1}(i-1)+A_{2}}{t}$ to show that $\mathbb{E}\left(N_{i, t}\right)=c_{i} t+o(t)$, where

$$
c_{i}=c_{i-1} p \frac{A_{1}(i-1)+A_{2}}{1+p\left(A_{1} i+A_{2}\right)} .
$$

It is easy to verify that the expression for $c_{i}$ as defined in (1) and (2) satisfies this recurrence relation.

We prove concentration for $N_{i, t}$ when $i \leq i_{f}$ by using a relaxation of Azuma-Hoeffding martingale techniques. The random variables $N_{i, t}$ do not a priori satisfy the $c$-Lipschitz condition: it is possible that a new node may fall into many overlapping regions of influence. Nevertheless, we will prove that deviation from the $c$-Lipschitz condition occurs with exponentially small probability. The following lemma gives a bound for $\left|N_{i, t+1}-N_{i, t}\right|$ which holds with extreme probability. 
Lemma 2.2. Wep the following inequality holds for all $0 \leq t \leq n-1$.

$$
\left|N_{i, t+1}-N_{i, t}\right| \leq 2\left(A_{1} i+A_{2}\right) \log ^{2} n, \text { for } 0 \leq i \leq t .
$$

Proof. Fix $t$, let $i, j \leq t$, and let $X_{j}(i, t)$ denote the indicator variable for the event that $v_{j}$ has degree $i$ at time $t$ and $v_{t+1}$ links to $v_{j}$. Thus,

$$
N_{i, t+1}-N_{i, t}=\sum_{j=1}^{t} X_{j}(i-1, t)-\sum_{j=1}^{t} X_{j}(i, t),
$$

and so

$$
\left|N_{i, t+1}-N_{i, t}\right| \leq \max \left(\sum_{j=1}^{t} X_{j}(i-1, t), \sum_{j=1}^{t} X_{j}(i, t)\right) .
$$

Let $Z_{j}(i, t)$ denote the indicator variable for the event that $v_{t+1}$ is chosen in the ball of volume $\left(A_{1} i+A_{2}\right) / t$ around node $v_{j}$. Clearly, if $X_{j}(i, t)=1$, then $Z_{j}(i, t)=1$ as well, so $X_{j}(i, t) \leq Z_{j}(i, t)$. Thus, to bound $\left|N_{i, t+1}-N_{i, t}\right|$ it suffices to bound the values of $Z(i, t)$, where

$$
Z(i, t)=\sum_{j=1}^{t} Z_{j}(i, t)
$$

The variables $Z_{j}(i, t)$ for $j=1, \ldots, t$ are pairwise independent. To see this, we can assume the position of $v_{t+1}$ to be fixed. Then, the value of $Z_{j}(i, t)$ depends only on the position of $v_{j}$. Since the position of each node is chosen independently and uniformly, the value of $Z_{j}(i, t)$ is independent from the value of any other $Z_{j^{\prime}}(i, t)$ where $j \neq j^{\prime}$. Therefore, $Z(i, t)$ is the sum of independent Bernouilli variables with probability of success equal to

$$
\mathbb{P}\left(Z_{j}(i, t)=1\right)=\frac{A_{1} i+A_{2}}{t} .
$$

Using Chernoff's inequalities (see, for instance Theorem 2.1 in [7]), we can show that wep $Z(i, t)<A_{1} i+A_{2}+\left(A_{1} i+A_{2}\right) \log ^{2} n<2\left(A_{1} i+A_{2}\right) \log ^{2} n$. Using these bounds, the proof now follows since by $(5)$,

$$
\left|N_{i, t+1}-N_{i, t}\right| \leq \max (Z(i-1, t), Z(i, t)) .
$$

Let us mention that Theorem 1.5 can be used to improve the upper bound for $\mid N_{i, n}-$ $N_{i, n-1} \mid$ to $O\left(\log ^{2} n\right)$ since the maximum change cannot be bigger than out-degree of vertex $v_{n}$.

To sketch the technique of the proof of Theorem 1.1, we consider $N_{0, t}$, the number of nodes of in-degree zero. We use the supermartingale method of Pittel et al. [10], as described in [11].

Lemma 2.3. Let $G_{0}, G_{1}, \ldots, G_{n}$ be a random graph process and $X_{t}$ a random variable determined by $G_{0}, G_{1}, \ldots, G_{t}, 0 \leq t \leq n$. Suppose that for some real $\beta$ and constants $\gamma_{i}$

$$
\mathbb{E}\left(X_{t}-X_{t-1} \mid G_{0}, G_{1}, \ldots, G_{t-1}\right)<\beta
$$

and

$$
\left|X_{t}-X_{t-1}-\beta\right| \leq \gamma_{i}
$$


for $1 \leq t \leq n$. Then for all $\alpha>0$,

$$
\mathbb{P}\left(\text { For some } t \text { with } 0 \leq t \leq n: X_{t}-X_{0} \geq t \beta+\alpha\right) \leq \exp \left(-\frac{\alpha^{2}}{2 \sum \gamma_{j}^{2}}\right) .
$$

Theorem 2.4. Wep for every $1 \leq t \leq n$, we have that

$$
N_{0, t}=\frac{t}{1+A_{2} p}+O\left(n^{1 / 2} \log ^{3} n\right)=c_{0} t+O\left(n^{1 / 2} \log ^{3} n\right) .
$$

Proof. We first transform $N_{0, t}$ into something close to a martingale. It provides some insight if we define real function $f(x)$ to model the behaviour of the scaled random variable $\frac{1}{n} N_{0, x n}$. If we presume that the changes in the function correspond to the expected changes of random variable (see (3)), we obtain the following differential equation

$$
f^{\prime}(x)=1-f(x) \frac{p A_{2}}{x}
$$

with the initial condition $f(0)=0$. The general solution of this equation can be put in the form

$$
f(x) x^{p A_{2}}-\frac{x^{1+p A_{2}}}{1+p A_{2}}=C .
$$

Consider the following real-valued function

$$
H(x, y)=x^{p A_{2}} y-\frac{x^{1+p A_{2}}}{1+p A_{2}}
$$

(note that we expect $H\left(t, N_{0, t}\right)$ to be close to zero). Let $\mathbf{w}_{t}=\left(t, N_{0, t}\right)$, and consider the sequence of random variables $\left(H\left(\mathbf{w}_{t}\right): 1 \leq i \leq n\right)$. The second-order partial derivatives of $H$ evaluated at $\mathbf{w}_{t}$ are all $O\left(t^{p A_{2}-1}\right)$. Therefore, we have

$$
H\left(\mathbf{w}_{t+1}\right)-H\left(\mathbf{w}_{t}\right)=\left(\mathbf{w}_{t+1}-\mathbf{w}_{t}\right) \cdot \operatorname{grad} H\left(\mathbf{w}_{t}\right)+O\left(t^{p A_{2}-1}\right),
$$

where "." denotes the scalar product and grad $H\left(\mathbf{w}_{t}\right)=\left(H_{x}\left(\mathbf{w}_{t}\right), H_{y}\left(\mathbf{w}_{t}\right)\right)$.

Observe that from our choice of $H$, we have that

$$
\mathbb{E}\left(\mathbf{w}_{t+1}-\mathbf{w}_{t} \mid G_{t}\right) \cdot \operatorname{grad} H\left(\mathbf{w}_{t}\right)=0 .
$$

Hence, taking the expectation of (7) conditional on $G_{t}$, we obtain that

$$
\mathbb{E}\left(H\left(\mathbf{w}_{t+1}\right)-H\left(\mathbf{w}_{t}\right) \mid G_{t}\right)=O\left(t^{p A_{2}-1}\right) .
$$

From (7), noting that

$$
\operatorname{grad} H\left(\mathbf{w}_{t}\right)=\left(p A_{2} t^{p A_{2}-1} N_{0, t}-t^{p A_{2}}, t^{p A_{2}}\right),
$$

and using Lemma 2.2 (and the comment after the lemma) to bound the change in $N_{0, t}$, we have that wep

$$
\left|H\left(\mathbf{w}_{t+1}\right)-H\left(\mathbf{w}_{t}\right)\right| \leq t^{p A_{2}} O\left(\log ^{2} n\right)+O\left(t^{p A_{2}}\right)=O\left(t^{p A_{2}} \log ^{2} n\right) .
$$

Now we may apply Lemma 2.3 to the sequence $\left(H\left(\mathbf{w}_{t}\right): 1 \leq i \leq n\right)$, and symmetrically to $\left(-H\left(\mathbf{w}_{t}\right): 1 \leq i \leq n\right)$, with $\alpha=n^{1 / 2+p A_{2}} \log ^{3} n, \beta=O\left(t^{p A_{2}-1}\right)$ and $\gamma_{t}=O\left(t^{p A_{2}} \log ^{2} n\right)$, to obtain that wep

$$
\left|H\left(\mathbf{w}_{t}\right)-H\left(\mathbf{w}_{0}\right)\right|=O\left(n^{1 / 2+p A_{2}} \log ^{3} n\right)
$$


for $1 \leq t \leq n$. As $H\left(\mathbf{w}_{0}\right)=0$, this implies from the definition (6) of the function $H$, that wep

$$
N_{0, t}=\frac{t}{1+p A_{2}}+O\left(n^{1 / 2} \log ^{3} n\right)
$$

for $1 \leq t \leq n$ which finishes the proof of the theorem.

We may repeat (recursively) the argument as in the proof of Theorem 2.4 for $N_{i, t}$ with $i \geq 1$. Since the expected change for $N_{i, t}$ is slightly different now (see (4)), we obtain our result by considering the following function:

$$
H(x, y)=x^{p\left(A_{1} i+A_{2}\right)} y-c_{i-1} \frac{p\left(A_{1}(i-1)+A_{2}\right)}{1+p\left(A_{1} i+A_{2}\right)} x^{1+p\left(A_{1} i+A_{2}\right)} .
$$

Using this function, we may show by similar arguments as in the case $i=0$ that wep

$$
N_{i, n}=c_{i} n+O\left(i n^{1 / 2} \log ^{3} n\right) .
$$

Thus, we obtain concentration for all degrees $i$ up to

$$
i_{f}=\left(\frac{n}{\log ^{8} n}\right)^{\frac{p A_{1}}{4 p A_{1}+2}},
$$

since

$$
\begin{aligned}
i_{f} n^{1 / 2} \log ^{3} n & =n^{\frac{3 p A_{1}+1}{4 p A_{1}+2}} \log ^{\frac{4 p A_{1}+6}{4 p A_{1}+2}} n \\
& =o\left(n^{\frac{3 p A_{1}+1}{4 p A_{1}+2}} \log ^{\frac{4 p A_{1}+6}{4 p A_{1}+2}+1} n\right) \\
& =o\left(i_{f}^{-\left(1+\frac{1}{p A_{1}}\right)} n\right)=o\left(c_{i_{f}} n\right) .
\end{aligned}
$$

2.2. Proof of Theorems $\mathbf{1 . 2}$ and 1.3. We present the proofs of the results on the in-degrees of individual nodes and the number of edges.

Proof of Theorem 1.2. To simplify notation let $\eta=A_{1} p, \nu=A_{2} p, \xi=A_{2} / A_{1}$. Let the node added at time-step $v$ be denoted $v$, and treat the current time-step (given as $n$ above) as $t$. Let $\mathbb{P}\left(d^{-}(v, t)=l\right)$ denote the distribution of in-degree of node $v$ at the end of time-step $t$.

The indicator variable $X(t+1)$ for an increase in $d^{-}(v, t)$ by receiving a link from $v_{t+1}$ is a Bernoulli random variable with parameter $p\left(A_{1} d^{-}(v, t)+A_{2}\right) / t$. Thus,

$$
\begin{aligned}
& \mathbb{P}\left(X(t+1)=0 \mid d^{-}(v, t)=j\right)=1-\frac{\eta j+\nu}{t}, \\
& \mathbb{P}\left(X(t+1)=1 \mid d^{-}(v, t)=j\right)=\frac{\eta j+\nu}{t} .
\end{aligned}
$$

Let $v, t$ be fixed, suppose $d^{-}(v, t)=l$ and let $\mathbf{T}=\left(T_{j}, j=1, \ldots, l\right)$ denote the timesteps $T_{j}$ (if any) at which the degree of $v$ changed. Let $\boldsymbol{\tau}=\left(\tau_{1}, \ldots, \tau_{l}\right)$ denote a particular value of $\mathbf{T}$, so that $\tau_{j}$ is the time-step at which $d^{-}\left(v, \tau_{j}\right)$ changed from $j-1$ to $j$. For $v<\tau \leq t$ let

$$
J=\left\{\boldsymbol{\tau}: \tau_{1}<\tau_{2}<\cdots<\tau_{l}\right\}
$$


be the sequences of possible transitions. Thus

$$
\mathbb{P}\left(d^{-}(v, t)=l\right)=\sum_{\boldsymbol{\tau} \in J} \mathbb{P}(\mathbf{T}=\boldsymbol{\tau}) .
$$

Let

$$
\Psi_{j}=\mathbb{P}\left(X(T)=0, \text { for all } \tau_{j}<T<\tau_{j+1}\right),
$$

with $\tau_{l}<T \leq \tau_{l+1}=t$ when $j=l$. If $\tau_{j+1}=\tau_{j}+1$ let $\Psi_{j}=1$. If $\tau_{j+1} \geq \tau_{j}+2$, then from (8) we have that

$$
\Psi_{j}=\prod_{\tau_{j}<T<\tau_{j+1}}\left(1-\frac{\eta j+\nu}{T}\right) .
$$

Define $\omega=\log t$. As $l \leq \sqrt{v} / \omega$, then $(\eta j+\nu) / t \leq(\eta l+\nu) / v=o(1)$ so that

$$
1-\frac{\eta j+\nu}{t}=e^{-\frac{\eta j+\nu}{t}-O\left(\frac{j^{2}}{t^{2}}\right)} .
$$

Let

$$
\delta(\tau, j)=j^{2} / \tau
$$

then

$$
\begin{aligned}
\Psi_{j} & =\exp \left(\left(-(\eta j+\nu) \sum_{\tau_{j}<T<\tau_{j+1}} \frac{1}{T}\right)-O\left(\delta\left(\tau_{j}, j\right)\right)\right) \\
& =\left(\frac{\tau_{j}}{\tau_{j+1}}\right)^{\eta j+\nu}\left(1+O\left(\delta\left(\tau_{j}, j\right)\right)\right) .
\end{aligned}
$$

For $0 \leq j \leq l-1$, let $\Phi_{j}(t+1)=\mathbb{P}\left(X(t+1)=1 \mid d^{-}(v, t)=j\right)$. Thus, from (9)

$$
\Phi_{j}(t+1)=\frac{\eta j+\nu}{t} .
$$

Let $\Phi_{j}=\Phi\left(\tau_{j+1}\right)$, and let $\Phi_{l}=1$. Let $F(\boldsymbol{\tau})$ denote $\mathbb{P}\left(d^{-}(v, t)=l\right.$ and $\left.\boldsymbol{\tau}\right)$. Let $\mathbb{P}\left(\boldsymbol{T}_{j}=\tau_{j} \mid \boldsymbol{T}_{j-1}=\tau_{j-1}\right)$ be the probability that the transition to $j$ occurs at $\tau_{j}$ given the transition to $j-1$ at $\tau_{j-1}$. Thus,

$$
F(\boldsymbol{\tau})=\Psi_{l} \prod_{j=1}^{l} \mathbb{P}\left(\boldsymbol{T}_{j}=\tau_{j} \mid \boldsymbol{T}_{j-1}=\tau_{j-1}\right)=\prod_{j=0}^{l} \Psi_{j} \Phi_{j} .
$$

Ignoring for the moment the multiplicative error terms, we see that $F(\boldsymbol{\tau})$ is given by

$$
\left(\frac{v}{\tau_{1}}\right)^{\nu} \frac{\nu}{\tau_{1}}\left(\frac{\tau_{1}}{\tau_{2}}\right)^{\eta+\nu} \frac{\eta+\nu}{\tau_{2}} \cdots\left(\frac{\tau_{l-1}}{\tau_{l}}\right)^{\eta(l-1)+\nu} \frac{\eta(l-1)+\nu}{\tau_{l}}\left(\frac{\tau_{l}}{t}\right)^{\eta l+\nu} .
$$

Recall that $\xi=\nu / \eta$. We cancel repeated values of $\tau_{j}$ to give

$$
F(\boldsymbol{\tau})=(1+O(\delta(t, l))) \frac{\Gamma(l+\xi)}{\Gamma(\xi)}\left(\frac{v}{t}\right)^{\nu} \prod_{j=1}^{l} \frac{\eta \tau_{j}^{\eta-1}}{t^{\eta}}\left(1+O\left(\delta\left(\tau_{j}, j\right)\right)\right)
$$

Thus,

$$
\mathbb{P}\left(d^{-}(v, t)=l\right)=(1+O(\delta(t, l))) \frac{\Gamma(l+\xi)}{\Gamma(\xi)}\left(\frac{v}{t}\right)^{\nu} P_{1}
$$


where

$$
P_{1}=\sum_{\boldsymbol{\tau} \in J} \prod_{j=1}^{l} \frac{\eta \tau_{j}^{\eta-1}}{t^{\eta}}\left(1+O\left(\delta\left(\tau_{j}, j\right)\right)\right) .
$$

For $b_{j} \geq 0$ we have that

$$
\left(b_{v}+\cdots+b_{t}\right)^{k}-\left(b_{v}{ }^{2}+\cdots+b_{t}{ }^{2}\right)\left(\begin{array}{l}
k \\
2
\end{array}\right)\left(b_{v}+\cdots+b_{t}\right)^{k-2} \leq k ! \sum_{i_{1}<\cdots<i_{k}} b_{i_{1}} \cdots b_{i_{k}} \leq\left(b_{v}+\cdots+b_{t}\right)^{k} .
$$

Replace the term $\delta\left(\tau_{j}, j\right)$ in $F(\boldsymbol{\tau})$ with $\delta\left(\tau_{j}, l\right)$ and let

$$
b_{\tau}=(1+O(\delta(\tau, l))) \frac{\eta \tau^{\eta-1}}{t^{\eta}}
$$

so that

$$
P_{1}=\frac{1}{l !}\left\{\left(b_{v}+\cdots+b_{t}\right)^{l}-O\left(l^{2}\right)\left(b_{v}{ }^{2}+\cdots+b_{t}{ }^{2}\right)\left(b_{v}+\cdots+b_{t}\right)^{l-2}\right\} .
$$

Using (10) and recalling that $l \leq \sqrt{v} / \omega$,

$$
\begin{aligned}
b_{v}+\cdots+b_{t} & =\sum_{v \leq \tau \leq t} \frac{\eta \tau^{\eta-1}}{t^{\eta}}(1+O(\delta(\tau, l))) \\
& =1-\left(\frac{v}{t}\right)^{\eta}\left(1-\left(\frac{v}{t}\right)^{\eta} O\left(\frac{l^{2}}{v}\right)\right) \\
& =1-\left(\frac{v}{t}\right)^{\eta}\left(1+O\left(\frac{1}{\omega}\right)\right) .
\end{aligned}
$$

An upper bound for $P_{1}$, and hence $\mathbb{P}\left(d^{-}(v, t)=l\right)$ follows.

For $1 \leq v \leq t(1-1 / \omega)$ and $l \leq \sqrt{v} / \omega$ we prove below that

$$
\sum_{\tau=v}^{t} b_{\tau}^{2}=O\left(\frac{1}{\omega l^{2}}\right)\left(\sum_{\tau=v}^{t} b_{\tau}\right)^{2}
$$

Thus, we obtain

$$
P_{1}=\left(1+O\left(\frac{1}{\omega}\right)\right) \frac{1}{l !}\left(1-\left(\frac{v}{t}\right)^{\eta}\left(1+O\left(\frac{1}{\omega}\right)\right)\right)^{l} .
$$

Inserting this estimate for $P_{1}$ into (11) completes the proof of Theorem 1.2 (a). As remarked in the previous paragraph, (13) is an upper bound for $P_{1}$ for any $l \leq \sqrt{v} / \omega$, which completes the proof of Theorem 1.2 (b).

Returning to the proof of (12), let

$$
g(v, t)= \begin{cases}\frac{\eta^{2}}{1-2 \eta}\left(\frac{1}{v}\left(\frac{v}{t}\right)^{2 \eta}-\frac{1}{t}\right) & \eta<1 / 2 \\ \frac{1}{4 t} \log (t / v) & \eta=1 / 2 \\ \frac{\eta^{2}}{2 \eta-1}\left(\frac{1}{t}-\frac{1}{v}\left(\frac{v}{t}\right)^{2 \eta}\right) & \eta>1 / 2 .\end{cases}
$$


Using $\delta=O(1 / \omega)$ we have that

$$
b_{v}{ }^{2}+\cdots+b_{t}{ }^{2}=\left(1+O\left(\frac{1}{\omega}\right)\right) g(v, t) .
$$

It follows by direct examination that $v g(v, t)=O(1)$. As $l \leq \sqrt{v} / \omega$, we have $l^{2} g(v, t)=$ $O\left(1 / \omega^{2}\right)$. However $\sum_{i} b_{i} \geq \Theta(1 / \omega)$ for $v \leq t(1-1 / \omega)$, and the result follows.

We now prove Theorem 1.2 (c). Let $X_{t}=d^{-}(v, t)$. By Markov's inequality, for $h>0$,

$$
\mathbb{P}\left(X_{t} \geq \alpha\right)=\mathbb{P}\left(e^{h X_{t}} \geq e^{h \alpha}\right) \leq e^{-h \alpha} \mathbb{E} e^{h X_{t}} .
$$

Let $Y_{t}$ be an indicator variable for the increase of in-degree of $v$ at time-step $t+1$, then $X_{t+1}=X_{t}+Y_{t}$ where

$$
\mathbb{P}\left(Y_{t}=1\right)=\frac{p\left(X_{t}+1\right)}{t+1}
$$

and

$$
\mathbb{E}\left(e^{h Y_{t}} \mid X_{t}\right)=1+\frac{p\left(X_{t}+1\right)}{t+1}\left(e^{h}-1\right) .
$$

Assume that $0<h \leq 1$ (proved below in (16)) so that $e^{h} \leq h+h^{2}$, then

$$
\begin{aligned}
\mathbb{E}\left(e^{h X_{t+1}}\right) & =\mathbb{E}\left(e^{h X_{t}} e^{h Y_{t}}\right) \\
& \leq \mathbb{E}\left(e^{h X_{t}} e^{\frac{p\left(X_{t}+1\right)}{t+1}\left(e^{h}-1\right)}\right) \\
& \leq e^{\frac{p h}{t+1}(1+h)} \mathbb{E}\left(e^{h X_{t}\left(1+\frac{p}{t+1}(1+h)\right)}\right) .
\end{aligned}
$$

Let $\epsilon=9 / \omega$, and let

$$
h=\frac{1}{\omega}\left(\frac{v}{t}\right)^{p(1+2 \epsilon)} .
$$

Let $h_{t}=h$ and for $v+1 \leq s \leq t$ define $h_{s-1}$ by

$$
h_{s-1}=h_{s}\left(1+\frac{p}{s}\left(1+h_{s}\right)\right) \text {, }
$$

so that

$$
h_{s}=h \prod_{\tau=s+1}^{t}\left(1+\frac{p}{\tau}\left(1+h_{\tau}\right)\right) .
$$

Let $\epsilon_{\tau}=\max \left(h_{\tau}: \tau=v, \ldots, h\right)$ and assume (proved below in (16)) that $\epsilon_{\tau}<\epsilon<1$.

Iterating expression (15) and noting that $\mathbb{E} e^{h_{v} X_{v}}=1$ as $X_{v}=0$, we have

$$
\mathbb{E} e^{h X_{t}} \leq \exp \left(p \sum_{s=v}^{t} \frac{h_{s}\left(1+h_{s}\right)}{s}\right) \leq \exp \left(p(1+\epsilon) \sum \frac{h_{s}}{s}\right) .
$$

However, as $1 / s+\cdots+1 / t \leq 1 / s+\log t / s$, we have

$$
h_{s} \leq h \exp \left(\sum_{\tau=s+1}^{t} \frac{p(1+\epsilon)}{\tau}\right) \leq h e^{2}\left(\frac{t}{s}\right)^{p(1+\epsilon)} \leq \frac{e^{2}}{\omega}<1,
$$

for $t \geq 9$. 
Thus

$$
\begin{aligned}
\mathbb{E} e^{h X_{t}} & \leq \exp \left(h p(1+\epsilon) e^{2} t^{p(1+\epsilon)} \sum_{s=v}^{t} \frac{1}{s^{1+p(1+\epsilon)}}\right) \\
& \leq \exp \left(h\left(\frac{t}{v}\right)^{p(1+\epsilon)} e^{2}\left(1+\frac{p(1+\epsilon)}{v}\right)\right) \\
& \leq \exp \left(\frac{e^{4}}{\omega}\left(\frac{v}{t}\right)^{\epsilon p}\right) \\
& =1+O\left(\frac{1}{\omega}\right) .
\end{aligned}
$$

Let $\alpha=K \omega^{2}(t / v)^{p}$. By (14) and (16) we have that

$$
\begin{aligned}
\mathbb{P}\left(X_{t} \geq \alpha\right) & =(1+o(1)) e^{-h \alpha} \\
& =O(1) \exp \left(-K \omega\left(\frac{v}{t}\right)^{2 p \epsilon}\right) \\
& =O\left(t^{-K e^{-18}}\right) .
\end{aligned}
$$

This completes the proof of item (c), and completes the proof of Theorem 1.2.

Proof of Theorem 1.3. We count the number of edges by counting the in-degree of nodes. Our approach is as follows: by Theorem 1.1 wep for $i \leq i_{f}$ the number of nodes $N_{i, n}$ of in-degree $i$ at time $n$ is concentrated.

Let $a$ be the solution of $(n / a)^{p A_{1}}=i_{f}$ and let $\omega^{\prime}=\left(K \log ^{2} n\right)^{1 /\left(p A_{1}\right)}$ be the solution of

$$
\left(\frac{n}{a \omega^{\prime}}\right)^{p A_{1}} K \log ^{2} n=\left(\frac{n}{a}\right)^{p A_{1}}
$$

where $K \geq 4 e^{18}$. From Theorem $1.2(\mathrm{c})$, with probability $1-O\left(n^{-3}\right)$ no node $v \geq a \omega^{\prime}$ has degree exceeding $i_{f}$. Let

$$
\mu(n)=\sum_{i \leq i_{f}} \mathbb{E} N_{i, n}=(1+o(1)) \sum_{i \leq i_{f}} N_{i, n}
$$

and let

$$
\lambda(n)=\sum_{j=1}^{a \omega^{\prime}} d^{-}\left(v_{j}, n\right)
$$


We prove, conditional on Theorem $1.2(\mathrm{c})$, that $\lambda(n)=o\left(m_{n}\right)$ and thus, the number of edges is concentrated around $m_{n}$. We have that for $p A_{1}<1$

$$
\begin{aligned}
\lambda(n) & =\sum_{j=1}^{a \omega^{\prime}} d^{-}\left(v_{j}, n\right) \\
& \leq K \omega^{2} \sum_{j=1}^{a \omega^{\prime}}\left(\frac{n}{j}\right)^{p A_{1}} \\
& =O\left(\omega^{2}\left(\frac{n}{a \omega^{\prime}}\right)^{p A_{1}} a \omega^{\prime}\right) \\
& =O\left(n\left(\frac{n}{a}\right)^{p A_{1}-1} \log ^{2 /\left(p A_{1}\right)} n\right) \\
& =O\left(n\left(\frac{n}{\log ^{8} n}\right)^{\left(p A_{1}-1\right) /\left(4 p A_{1}+2\right)} \log ^{2 /\left(p A_{1}\right)} n\right) \\
& =O\left(n^{\left(5 p A_{1}+1\right) /\left(4 p A_{1}+2\right)} \log ^{2 /\left(p A_{1}\right)} n\right) \\
& =O(n) .
\end{aligned}
$$

However, $\mu(n) \geq c n$ for some constant $c>0$ so $\lambda(n)=o(\mu(n))$, and the assertion follows.

2.3. Proof of Theorems $\mathbf{1 . 4}$ and 1.5. We now give the proofs of the results on outdegrees in the SPA model.

Proof of Theorem 1.4. Partition the interval $[0,1]$ into $\left\lceil 2\left(n / A_{2}\right)^{1 / m}\right\rceil$ subintervals of the equal length. Hence, the unit hypercube is partitioned into

$$
h=2^{m} n / A_{2}+O\left(n^{(m-1) / m}\right)=(1+o(1)) 2^{m} n / A_{2}
$$

identical hypercubes. (We expect each hypercube to contain a constant number of nodes.) We will show that aas there is a hypercube containing $\frac{\log n}{\log \log n}$ nodes.

Fix $c \in \mathbb{R}$ and suppose that

$$
k=k(n)=\frac{\log n}{\log \log n}\left(1+c_{n}\right)
$$

such that

$$
\lim _{n \rightarrow \infty}(k+1 / 2)\left(\log k+m \log 2-\log A_{2}-1\right)=\log n+c .
$$

Note that $k=\frac{\log n}{\log \log n}(1+O(\log \log \log n / \log \log n))=(1+o(1)) \frac{\log n}{\log \log n}$. 
The probability $q$ that any fixed hypercube contains exactly $k$ nodes is equal to

$$
\begin{aligned}
q & =\left(\begin{array}{l}
n \\
k
\end{array}\right)\left(\frac{1}{h}\right)^{k}\left(1-\frac{1}{h}\right)^{n-k} \\
& =(1+o(1)) \frac{n^{k}}{k !}\left(\frac{A_{2}}{2^{m} n}\right)^{k} \exp \left(-\frac{A_{2}}{2^{m}}\right) \\
& =(1+o(1)) \frac{1}{k !}\left(\frac{A_{2}}{2^{m}}\right)^{k} \exp \left(-\frac{A_{2}}{2^{m}}\right) .
\end{aligned}
$$

Using Stirling's formula $k !=(1+o(1)) \sqrt{2 \pi k}(k / e)^{k}$, we obtain that

$$
\begin{aligned}
q & =(1+o(1)) \sqrt{\frac{2^{m}}{2 \pi A_{2}}}\left(\frac{e A_{2}}{2^{m} k}\right)^{k+1 / 2} \exp \left(-\frac{A_{2}}{2^{m}}-\frac{1}{2}\right) \\
& =(1+o(1)) \sqrt{\frac{2^{m}}{2 \pi A_{2}}} \exp \left(-(k+1 / 2)\left(\log k+m \log 2-\log A_{2}-1\right)-\frac{A_{2}}{2^{m}}-\frac{1}{2}\right) \\
& =(1+o(1)) \sqrt{\frac{2^{m}}{2 \pi A_{2}}} \exp \left(-\log n-c-\frac{A_{2}}{2^{m}}-\frac{1}{2}\right) \\
& =(1+o(1)) \frac{1}{n} \sqrt{\frac{2^{m}}{2 \pi A_{2}}} \exp \left(-c-\frac{A_{2}}{2^{m}}-\frac{1}{2}\right) .
\end{aligned}
$$

Thus, the expected number of hypercubes with exactly $k$ nodes is tending to

$$
\lambda=h q=\frac{1}{\sqrt{2 \pi}}\left(\frac{2^{m}}{A_{2}}\right)^{3 / 2} \exp \left(-c-\frac{A_{2}}{2^{m}}-\frac{1}{2}\right) .
$$

Now, let $A_{i}(1 \leq i \leq h)$ denote an event that $i$ th hypercube contains exactly $k$ nodes, and let $S_{h}=\sum_{i=1}^{h} I_{A_{i}}$ be the number of events which actually occur ( $S_{h}$ is a random variable). Finally, let

$$
B_{l}^{h}=\sum_{1 \leq j_{1}<\cdots<j_{l} \leq h} \mathbb{P}\left(\bigcap_{i=1}^{l} A_{j_{i}}\right) .
$$

We already showed that $\lim _{h \rightarrow \infty} B_{1}^{h}=\lambda$. It is also not difficult to see that for a fixed value of $l$ that

$$
\lim _{h \rightarrow \infty} B_{l}^{h}=\frac{\lambda^{l}}{l !}
$$

Therefore, $S_{h}$ is tending to a random variable with Poisson distribution; that is,

$$
\lim _{h \rightarrow \infty} \mathbb{P}\left(S_{h}=l\right)=\frac{\lambda^{l}}{l !} e^{-\lambda} .
$$

In particular,

$$
\lim _{h \rightarrow \infty} \mathbb{P}\left(S_{h}=0\right)=e^{-\lambda} .
$$

Since $c \rightarrow-\infty$ for $k=k_{0}=\frac{\log n}{\log \log n}$, aas there is a hypercube $K$ with $k_{0}$ points.

Since all nodes have the volume of the ball of influence at least $A_{2} / n$ during the whole process up to time $n$ (deterministically), the last node $v$ added to $K$ falls into 
balls of influence of all other nodes inside $K$ (observe that the volume of $K$ is at most $2^{-m} A_{2} / n$ so this holds even if $v$ lies on the boundary of $\left.K\right)$. Thus, $\mathbb{E} \operatorname{deg}^{+}(v, n) \geq p k_{0}$.

To finish the proof, we use the fact that a sum of independent random variables with large enough expected value is not too far from its mean (see, for example, Theorem 2.8 in [7]). From this it follows that, if $\epsilon \leq 3 / 2$, then

$$
\mathbb{P}\left(\left|\operatorname{deg}^{+}(v, n)-\mathbb{E} \operatorname{deg}^{+}(v, n)\right| \geq \epsilon \mathbb{E} \operatorname{deg}^{+}(v, n)\right) \leq 2 \exp \left(-\frac{\epsilon^{2}}{3} \mathbb{E} \operatorname{deg}^{+}(v, n)\right) .
$$

Setting

$$
\epsilon=1 / \sqrt[3]{\mathbb{E} \operatorname{deg}^{+}\left(v_{i}, n\right)}
$$

we obtain that aas

$$
\operatorname{deg}^{+}\left(v_{i}, n\right)=(1+O(\epsilon)) \mathbb{E} \operatorname{deg}^{+}\left(v_{i}, n\right),
$$

and the assertion follows.

Proof of Theorem 1.5. Since the node $v_{n}$ is chosen uar from the unit hypercube (note that the history of the process does not affect this distribution) with the torus metric, without loss of generality, we may assume that $v_{n}$ lies in the centre of the hypercube. For $1 \leq i<n$, let $X_{i}$ denote the indicator random variable of the event that $v_{i}$ lies in the ball around $v_{n}$ (or vice versa) with volume

$$
\alpha=2 i^{-p A_{1}} n^{p A_{1}-1} \log ^{2} n .
$$

By Theorem 1.2 (c), we have that aas

$$
d^{-}\left(v_{i}, n\right) \leq(n / i)^{p A_{1}} \log ^{2} n,
$$

for all $i \in[n]$. Thus, aas for all $i \in[n-1], X_{i}=0$ implies that $v_{n}$ is not in the influence region of $v_{i}$ and there is no directed edge from $v_{n}$ to $v_{i}$. Therefore, aas we have that

$$
\operatorname{deg}^{+}\left(v_{n}, n\right) \leq \sum_{i=1}^{n-1} X_{i} .
$$

Since

$$
\begin{aligned}
\mathbb{E}\left(\sum_{i=1}^{n-1} X_{i}\right) & =\sum_{i=1}^{n-1} O\left(i^{-p A_{1}} n^{p A_{1}-1} \log ^{2} n\right) \\
& =O\left(n^{p A_{1}-1} \log ^{2} n \sum_{i=1}^{n-1} i^{-p A_{1}}\right) \\
& =O\left(\log ^{2} n\right),
\end{aligned}
$$

the assertion follows from Chernoff bound (see (17)). 
2.4. Proof of Theorems 1.6 and 1.7. To prove Theorem 1.6 we need the following lemma whose (straightforward) proof is omitted.

Lemma 2.5. Let $u$ be chosen uar from a ball with centre $v$ and volume $\alpha$. If $X$ is the distance between $u$ and $v$, according to the torus metric as defined in the introduction, then

$$
\mathbb{E}(X)=\left(\frac{m}{2(m+1)}\right) \alpha^{1 / m} .
$$

Proof of Theorem 1.6. Define

$$
Z_{j, t}= \begin{cases}L\left(v_{t}, v_{j}\right) & \text { if }\left(v_{t}, v_{j}\right) \in E_{t} \\ 0 & \text { else. }\end{cases}
$$

Then $L_{t}=\sum_{j=1}^{t-1} Z_{j, t}$. Let $B_{t, j}$ be the event that $\left(v_{t}, v_{j}\right) \in E_{t}$. Then using Lemma 2.5 we have that

$$
\begin{aligned}
\mathbb{E}\left(Z_{j, t+1} \mid G_{t}\right) & =\mathbb{P}\left(B_{t+1, j}\right) \mathbb{E}\left(Z_{j, t+1} \mid G_{t}, B_{t+1, j}\right)+\mathbb{P}\left(\overline{B_{t+1, j}}\right) \mathbb{E}\left(Z_{j, t+1} \mid G_{t}, \overline{B_{t+1, j}}\right) \\
& =\mathbb{P}\left(B_{t+1, j}\right) \mathbb{E}\left(L\left(\left(v_{t+1}, v_{j}\right) \mid G_{t}\right)\right. \\
& =\left(p \frac{A_{1} d^{-}\left(v_{j}, t\right)+A_{2}}{t}\right)\left(\frac{m}{2(m+1)}\right)\left(\frac{A_{1} d^{-}\left(v_{j}, t\right)+A_{2}}{t}\right)^{1 / m} \\
& =\frac{p m}{2(m+1)}\left(\frac{A_{1} d^{-}\left(v_{j}, t\right)+A_{2}}{t}\right)^{1+1 / m},
\end{aligned}
$$

where the second last equality follows by Lemma 2.5 and the definition of the model, and the second equality follows from the definition of $Z_{j, t+1}$. Thus

$$
\mathbb{E}\left(L_{t+1} \mid G_{t}\right)=\sum_{k=0}^{t} \sum_{\left\{j \mid d^{-}\left(v_{j}, t\right)=k\right\}} \mathbb{E}\left(Z_{j, t+1} \mid G_{t}\right)=\frac{p m}{2(m+1)} \sum_{k=0}^{t}\left(\frac{A_{1} k+A_{2}}{t}\right)^{1+1 / m} N_{k, t} .
$$

Taking expectations on both sides, and using that $\mathbb{E}\left(N_{k, t}\right)=(1+o(1)) c k^{-\left(1+\frac{1}{p A_{1}}\right)} t$ (see Theorem 1.1), we have that

$$
\begin{aligned}
\mathbb{E}\left(L_{t+1}\right) & =\frac{p m}{2(m+1)} \sum_{k=0}^{t}\left(\frac{A_{1} k+A_{2}}{t}\right)^{1+1 / m} \mathbb{E}\left(N_{k, t}\right) \\
& =(1+o(1)) \frac{p m}{2(m+1) t^{1 / m}} \sum_{k=0}^{t}\left(A_{1} k+A_{2}\right)^{1+1 / m} c k^{-\left(1+1 /\left(p A_{1}\right)\right)} \\
& =(1+o(1)) \frac{p A_{1}^{1+1 / m} c m}{2(m+1) t^{1 / m}} \int_{0}^{t} x^{1 / m-1 /\left(p A_{1}\right)} d x \\
& = \begin{cases}\Theta\left(t^{-} \min \{1 / m)\right. & \text { if } 1 / m<1 /\left(p A_{1}\right)-1 \\
\Theta\left(t^{-} \min \{1 / m \log n)\right. & \text { if } 1 / m=1 /\left(p A_{1}\right)-1 \\
\Theta\left(t^{-} \min \{1 / m)\right. & \text { if } 1 / m>1 /\left(p A_{1}\right)-1 .\end{cases}
\end{aligned}
$$

The last step is justified since it can be shown that the $o(1)$ term in the integrand is in fact $O\left(x^{-\epsilon}\right)$ for some $\epsilon>0$. 
Proof of Theorem 1.7. With the same notation as in the proof of Theorem 1.6 and using Lemma 2.5, we have that

$$
\begin{aligned}
\mathbb{E}\left(Z_{j, t+1} \mid G_{t}\right) & =\mathbb{P}\left(B_{j, t+1}\right) \mathbb{E}\left(L\left(v_{t+1}, v_{j}\right) \mid B_{j, t+1}\right) \\
& =\frac{p m}{2(m+1)}\left(\frac{d}{t}\right)^{1+1 / m} .
\end{aligned}
$$

Taking the expectation, we see that the terms $\mathbb{E}\left(Z_{j, t+1}\right)$ are equal for all $j$, and thus,

$$
\mathbb{E}\left(L_{t+1}\right)=t \mathbb{E}\left(Z_{j, t+1}\right)=\Theta\left(t^{-1 / m}\right)
$$

\section{Generalizations}

Several variants of the SPA model may be proposed. In each variant, it would be interesting to pursue a rigorous analysis of both the in-degree and out-degree distribution, and small world properties. One such variation is the Generalized SPA (or GSPA) model, which allows more control of the out-degree. In the GSPA model, nodes are distributed on the hypercube as in the SPA model, but now receive two regions of influence. Each node $v$ at time-step $t$ is assigned both an in-degree influence region with volume

$$
\frac{A_{i}+B_{i} d^{-}(v, t)}{t},
$$

where $A_{i}$ and $B_{i}$ are non-negative constants and $d^{-}(v, t)$ is the indegree of $v$ at time $t$, and an out-degree influence region with volume

$$
\frac{A_{o}+B_{o} d^{+}(v, t)}{t}
$$

where $A_{o}$ and $B_{o}$ are non-negative constants and $d^{+}(v, t)$ is the out-degree of $v$ at time $t$.

Edges are now added with probability $p$ between any pair of nodes whose regions interact by a pre-determined rule. An important difference with the SPA model is that at every time step all nodes can potentially receive out- and in-edges. This implies that graphs generated by the GSPA model can have cycles, and edges that go from younger to older nodes.

We describe three rules for the generation of edges.

Intersection rule. If the in-degree influence region of node $v$ has a nonempty intersection with the out-degree influence region of node $u$, then the directed edge $(u, v)$ is added.

Disjunction rule. If node $u$ is contained in the in-degree influence region of $v$, or node $v$ is contained in the out-degree influence region of $u$, then the directed edge $(u, v)$ is added.

Conjunction rule. If node $u$ is contained in the in-degree influence region of $v$, and node $v$ is contained in the out-degree influence region of $u$, then the directed edge $(u, v)$ is added. 
One of the rules is chosen (or some combination of them, depending on the motivating application), and edges are added according to the rules. Observe that the disjunction rule is the closest to the SPA model with $A_{o}=B_{o}=0$. Note that edges may well be added between pairs of older nodes in a given time-step, not just between the new node and the older nodes. The SPA model also has a fairly small bound $B$ on the out-degree with high probability (see Theorem 1.5). This implies that the graphs so generated have treewidth at most $B$ with high probability, which does not accurately model the large treewidth observed in the web graph (see [1]). The GSPA model is easily converted into an undirected model. In this model, there is an influence region based on degree. An edge is added between two nodes according to an overlap rule. The overlap rules above are easily modified to the undirected case.

\section{REFERENCES}

[1] W. Aiello, F.R.K. Chung, L. Lu, A random graph model for massive graphs, Experimental Mathematics 10 (2001) 53-66.

[2] A. Bonato, A Course on the Web Graph, American Mathematical Society, Providence, Rhode Island, 2008.

[3] F.R.K. Chung, L. Lu, Complex Graphs and Networks, American Mathematical Society, Providence, Rhode Island, 2006.

[4] C. Cooper, The age specific degree distribution of web-graphs, Combinatorics Probability and Computing 15 (2006) 637-661.

[5] A. Flaxman, A.M. Frieze, J. Vera, A geometric preferential attachment model of networks, Internet Mathematics 3 (2006) 187-205.

[6] A. Flaxman, A.M. Frieze, J. Vera, A geometric preferential attachment model of networks II, In: Proceedings of WAW'07, 2007.

[7] S. Janson, T. Łuczak, A. Ruciński, Random Graphs, Wiley, New York, 2000.

[8] F. Menczer, Lexical and semantic clustering by web links, JASIST 55(14) (2004), 1261-1269.

[9] M. Penrose, Random Geometric Graphs, Oxford University Press, Oxford, 2003.

[10] B. Pittel, J. Spencer, N. Wormald, Sudden emergence of a giant $k$-core in a random graph, Journal of Combinatorial Theory, Series B 67 (1996) 111-151.

[11] N. Wormald, The differential equation method for random graph processes and greedy algorithms, In: Lectures on Approximation and Randomized Algorithms, eds. M. Karoński and H. J. Prömel, PWN, Warsaw, (1999) 73-155. 